\title{
Evolución de un Programa de Formación en Competencias Genéricas
}

\author{
Rodolfo Schmal \\ Universidad de Talca, Facultad de Economía y Negocios, Escuela de Ingeniería en Informática \\ Empresarial, Talca -Chile (e-mail: rschmal@utalca.cl)
}

Recibido Mar. 6, 2015; Aceptado May. 14, 2015; Versión final Jun. 16, 2015, Publicado Dic. 2015

\begin{abstract}
Resumen
El objetivo de este trabajo es examinar la evolución que ha experimentado un modelo curricular basado en competencias que desde hace una década se encuentra implementando la Universidad de Talca en Chile. Uno de los componentes de dicho modelo es el programa de formación fundamental (PFF) orientado al desarrollo de competencias genéricas. Primero se dan algunos antecedentes que explican la existencia del PFF, seguido del análisis de cada una de sus versiones que ha tenido hasta la fecha, siendo la versión PFF 3.0 la más reciente. Lo más relevante del nuevo PFF (3.0) es que reduce y clarifica significativamente las competencias que compromete desarrollar, y redistribuye sus módulos dentro del plan de estudios. . Se concluye con algunas propuestas de acción orientadas a la consolidación del modelo curricular basado en competencias.
\end{abstract}

Palabras clave: competencias genéricas, desarrollo curricular, currículum por competencias

\section{Evolution of a Training Program in Generic Skills}

\begin{abstract}
The objective of this paper is to analyze the changes that a competency-based curriculum model that the University of Talca in Chile has been implementing for the last ten years. One of the components of such a model is the Fundamental Training Program (PFF) aimed toward developing generic skills. First, some general in formation that explain the existence of de PFF, followed by an analysis of the changes that the model has experienced (being PFF 3.0 the latest version. The most relevant aspect of PFF version 3.0 is that it significantly reduces and clarified the competences that must be developed, and reorganize the different modules within the study program. The paper concludes with some proposal oriented to consolidate the competency-based curriculum model.
\end{abstract}

Keywords: generic skills, curriculum development, curriculum competency 


\section{INTRODUCCIÓN}

Desde el año 2005, la Universidad de Talca, universidad regional estatal chilena, se encuentra implementando un modelo curricular orientado al desarrollo de competencias en todas las carreras de pregrado que imparte. Este modelo curricular se diseñó en base a 3 componentes o programas: un programa de formación fundamental (PFF), un programa de formación básica (PFB) y un programa de formación disciplinaria (PFD). Este trabajo se centra en el primer programa, el PFF, cuyo objetivo es desarrollar competencias genéricas o transversales, que se relacionan con aspectos no cognitivos, en particular, con el saber ser, el saber estar, y el saber actuar (UTalca, 2005; Hawes y Corvalán, 2005; Faúndez et al., 2009; Moyano et al., 2012).

Por la relevancia de la transformación curricular, a 5 años de su inicio, la comunidad académica -docentes y estudiantes- fue invitada a reflexionar en torno al diseño e implementación del PFF 1.0. Fruto de esta reflexión colectiva surge un PFF 2.0, que recoge la experiencia acumulada en el marco de una política de mejoramiento continuo en una realidad de gran dinamismo, tanto en la oferta de profesionales, como en la demanda de éstos por parte de las empresas y la sociedad misma. Posteriormente, en el año 2014, con miras a consolidar el modelo curricular basado en competencias, tiene lugar una nueva conversación entre los distintos actores involucrados -alumnos, profesores, empleadores, profesionales, ejecutivos, directivos), para dar origen a una tercera versión del PFF, el PFF 3.0, que inicia su andadura el 2015.

El objetivo de este trabajo es presentar la evolución del PFF en sus avances e insuficiencias en la organización, diseño e implementación del PFF, que desde su origen ha buscado subsanar las deficiencias de los alumnos que ingresan a la universidad en aquellas competencias que la sociedad y el mercado laboral están demandando con mucha fuerza. Esta presentación se efectúa desde la experiencia vivida en una de las carreras de Ingeniería, la de Ingeniería en Informática Empresarial (IIE), dada la relevancia que las empresas están asignando a las competencias genéricas de los egresados (Sicilia, 2009; Schmal, 2012; Yanaze y De Deus, 2014; Savaneviciene et al., 2014). Para el logro de este objetivo, el trabajo se ha estructurado como sigue. En primer lugar, se presentan los antecedentes que explican la instalación de un programa de formación en competencias transversales en la Universidad de Talca. Luego se describen los recursos metodológicos ocupados. Posteriormente se analizan y evalúan las características del PFF en sus tres versiones a partir de la aplicación de encuestas y la realización de talleres con los actores implicados. Luego se presentan los resultados que arroja un análisis comparativo entre las distintas versiones, para finalmente dar a conocer las conclusiones y recomendaciones que se desprenden.

En las últimas décadas se ha producido un sostenido incremento en la cobertura de la educación superior chilena, desde menos del $20 \%$ a más del $40 \%$ entre los jóvenes en edad de cursar estudios superiores. Este aumento se ha concentrado en los jóvenes de familias pertenecientes a los quintiles de más bajos ingresos (Olavarría y Allende, 2013). En el caso particular de la Universidad de Talca, se destaca que el $90 \%$ de su matrícula corresponde a alumnos de los tres primeros quintiles, provenientes en su mayoría de familias cuyos padres no son profesionales, y con ingresos mensuales inferiores a los US $\$ 600$.

Esta expansión en la educación superior, tiene su origen en la desregulación experimentada por el sector desde la década de 1981 en un contexto dado por un régimen político dictatorial y un modelo económico neoliberal. Se debe señalar que hasta los años 80 , las universidades eran altamente selectivas, y quienes ingresaban a ellas, por lo general, eran alumnos de familias con altos ingresos, quienes usufructuaban de estudios superiores prácticamente gratuitos (Carmona et al., 2014). En la actualidad, en cambio, una alta proporción de la matrícula universitaria proviene de familias con bajo capital social-cultural y de bajos ingresos. A ello se agrega que la universidad dejó de ser gratuita, además de ser su costo uno de los más altos a nivel mundial en relación al ingreso per cápita. Dada la insuficiencia del sistema de becas destinado a paliar el impacto del costo de la matrícula universitaria en el presupuesto familiar, la masificación universitaria ha sido posible sobre la base del endeudamiento de las familias (Brunner, 2009; Molina, 2013). Lo anterior se explica porque el modelo educativo instalado en Chile ha considerado la educación universitaria como un bien de consumo antes que como un bien público.

Al mismo tiempo, estas han sido décadas de un progreso científico-tecnológico sin precedentes, que está incidiendo tanto en el ámbito productivo como en el de la interacción social. De una sociedad basada en la producción de bienes, a una basada en la producción de servicios; de una sociedad que a sus profesionales les exigía conocimientos específicos perdurables en el tiempo, a una sociedad que está planteando exigencias que se relacionan más con el comportamiento que con los conocimientos, en un mundo inestable, cambiante (Corominas, 2001; Castells, 2005; Silva, 2007; Villarroel y Bruna, 2014). De esta nueva realidad, a la universidad y a las Escuelas de Ingeniería en particular, se le está exigiendo que se haga responsable. Fruto de un diagnóstico efectuado en los primeros años del presente milenio, se observó que los estudiantes seleccionados en primer año, en todas las carreras de pregrado de la Universidad de Talca, 
no contaban con los recursos personales que les permitieran enfrentar con éxito sus estudios universitarios, ya sea porque no contaban con las competencias de análisis crítico, de síntesis, comunicación y trabajo en equipo, como por poseer una escasa comprensión de los procesos sociales, económicos, políticos y culturales que están teniendo lugar en la sociedad contemporánea (Faúndez et al., 2009).

En este marco, la Universidad de Talca, en su carácter de universidad estatal y regional, asumió la responsabilidad de diseñar e implementar un modelo educativo que fuera más allá de la formación profesional centrada en el conocimiento de contenidos y competencias especializadas. Esto, con miras a la formación de personas capaces de contribuir eficaz y eficientemente, como profesionales y ciudadanos, al desarrollo político, económico, social y cultural del espacio en que se insertan. Lo señalado se complementa con el interés de la universidad porque los estudiantes que ingresan puedan terminar exitosamente sus estudios, disminuyendo las tasas de deserción y repitencia. (Rojas y Tarud, 2004). En base a lo expuesto la universidad reemplazó el modelo educativo tradicional por un modelo educativo basado en competencias, decisión que involucró a la totalidad de los programas de docencia de pregrado (carreras) existentes (Moyano et al, 2012). Luego de la transformación curricular que tuvo lugar, actualmente la universidad se encuentra transitando hacia la consolidación de este nuevo modelo educativo.

El concepto de competencia tiene un origen empresarial, cuando su definición se limitaba al saber hacer. Por ello, para algunos autores (Peluffo y Knust, 2009; Ruiz, 2009; Martí et al., 2014), el modelo de educación basado en el desarrollo de competencias, representa un paso más hacia la mercantilización de la universidad, cuyo punto de partida fijan en el proceso de Bolonia (González et al., 2004; Villa y Poblete, 2011). Sin embargo, actualmente la competencia no se limita al saber hacer, sino que se extiende al saber ser, estar y actuar, dándole una connotación que va más allá de la empresa, razón por la cual la concepción actual de competencia es una importante contribución a la construcción de una sociedad más democrática, solidaria y tolerante (Aristimuño, 2004; Hawes y Corvalán, 2005; Yániz, 2008; Salas, 2014; Villarroel y Bruna, 2014). En este trabajo se asume la postura de estos últimos, entendiéndose la competencia como la movilización, la puesta en acción, de un conjunto de capacidades -cognitivas, procedimentales y actitudinales- que se deben integrar en contextos específicos (Tardif, 2008). De allí que el diseño y la implementación de modelos curriculares basados en competencias ha implicado una revisión, tanto de las concepciones pedagógicas que lo sustentan, como de la vinculación con la sociedad, de los objetivos que se persiguen, de las relaciones interdisciplinarias, de las metodologías de enseñanza-aprendizaje y de los sistemas de evaluación (Alonso et al., 2013; Palominos et al., 2014).

Las competencias genéricas tienden a definirse por contraste con las competencias específicas, y por tanto, como aquellas que rebasan los límites de una disciplina, necesarias para iun desempeño eficaz y eficiente, más allá de una profesión en particular, y relevantes para desempeñarse en distintos contextos y para el aprendizaje a lo largo de la vida (Sicilia, 2009; Kallioinen, 2010). Yaniz (2008) las define de similar manera, pero enfatizando su relación con el desarrollo personal y la formación ciudadana. Por su parte, Savaneviciene et al. (2014) expresa que las competencias genéricas son aquellas que permiten actuar exitosamente en contextos: a) con insuficiente información y/o donde no existen reglas claramente definidas; b) con dosis de incertidumbre en las consecuencias de las decisiones, susceptibles de generar estress; y/o c) con horizontes de tiempos de actuación indefinidos. En la actualidad, para un eficiente trabajo profesional, se observa un creciente interés por las competencias genéricas para abordar las distintas demandas de los mercados y de la sociedad, más allá de los conocimientos técnicos, las competencias específicas propias de cada profesión.

Para el desarrollo, tanto de las competencias genéricas como específicas, de entre las diversas opciones existentes en el campo de las competencias (Mertens, 1996; Tobón, 2008), la Universidad de Talca optó por un enfoque socioconstructivista, el cual se centra en el recorrido para la construcción de la competencia, de allí su denominación (constructivista) y su carácter social, asumiendo que el desempeño de una organización se da por un trabajo colaborativo de personas competentes, antes que por la competencia de una persona en particular. Bajo este enfoque se destaca la importancia que tienen tanto las actividades de aprendizaje conducidas por los docentes, como el contexto en que se realizan y los saberes que trae consigo el estudiante en la construcción o desarrollo de la competencia (Jonnaert, 2001; Yániz, 2008).

El resultado de este enfoque fue una estructura curricular (UTalca, 2005): a) organizada en base a módulos valorados en créditos SCT(Sistema de Créditos Transferibles), equivalentes a los créditos ECTS (European Credit Transfer System), donde cada crédito representa 27 horas cronológicas de trabajo académico de un estudiante, el que incluye clases en aula, trabajos prácticos, seminarios, estudio individual en la casa o en biblioteca y pruebas u otras actividades de evaluación; b) planificada en base a cursos o módulos a ser impartidos en un semestre académico (18 semanas) o en un año académico (36 semanas); c) conformada en base a un máximo de créditos SCT recomendables de 30 por semestre y 60 por año académico. Dentro del creditaje SCT de cada módulo al menos dos tercios de los créditos deben asociarse a las actividades de 
aprendizaje autónomas fuera de aula, según lo requiera el nivel de desarrollo de las competencias respectivas; los créditos restantes se asignan a las actividades presenciales de los alumnos en aula insertas en sesiones de 60 minutos; d) desarrollada en 2 etapas: la primera, conducente a la obtención del grado de licenciado (240 SCT), y la segunda, a la obtención del título profesional (60 SCT), que se puede extender en 60 SCT adicionales; y e) estructurada en base a 3 componentes: de formación fundamental, de formación básica y de formación profesional, disciplinaria o específica, donde el primero está destinado a desarrollar las competencias genéricas, también llamadas transversales, de carácter instrumental para el trabajo académico, de desarrollo personal y ciudadana (Hawes y Corvalán, 2005; Moyano et al., 2012).

A diferencia de otros modelos curriculares donde las competencias fundamentales (genéricas o transversales) se desarrollan junto con las competencias básicas y/o disciplinares (Zúñiga et al., 2008; Villarroel y Bruna, 2014; Yániz, 2015), la Universidad de Talca, escogió un modelo curricular cuyo componente de formación fundamental, en su primera versión, asumiera la responsabilidad de desarrollar las competencias genéricas de forma tal que los alumnos estén en condiciones de ponerlas en acción en los módulos de los otros componentes (de formación básica y disciplinar). Esta opción fue escogida en consideración a los déficits que en materia de competencias genéricas presenta gran parte del alumnado que ingresa a la universidad.

\section{METODOLOGÍA}

Dado el objetivo perseguido, examinar la evolución de un programa de formación en competencias genéricas, la metodología de trabajo empleada fue de carácter empírico descriptivo basado en la observación, el análisis documental, entrevistas semiestructuradas y encuestas (Montero y León, 2002; Hernández et al., 2003). Para el levantamiento del perfil de egreso de los egresados, expresado en términos de competencias, durante el año 2003 se realizó un taller DACUM (Developing a Curriculum) (Norton, 1987), para cada una de las 19 carreras impartidas por la Universidad de Talca. Cada uno de estos talleres tuvo una duración de dos días con la participación de profesionales de las correspondientes carreras, junto con empresarios/empleadores. Durante el desarrollo de estos talleres emergieron tanto las competencias específicas esperadas en los egresados de cada profesión, como las competencias genéricas o transversales demandadas a la totalidad de las profesiones. Posteriormente, las competencias genéricas fueron clasificadas en competencias instrumentales, interpersonales y ciudadanas, dando origen a la primera versión del PFF (Hawes y Corvalán 2005).

El análisis de las versiones siguientes se realizó con: a) la participación del autor en talleres que acompañaron el proceso bajo la responsabilidad del Centro para la Innovación y la Calidad de la Docencia (CICAD), dependiente de la Vicerrectoría de Docencia de Pregrado de la Universidad de Talca; b) entrevistas con responsables del PFF en CICAD; y c) la lectura y análisis de las resoluciones adoptadas por la universidad. Para la evaluación del PFF 1.0 se aplicó una encuesta a una muestra de estudiantes de la carrera de IIE cuyos resultados sirvieron de insumo para ser analizados en jornadas de reflexión con los profesores que imparten clases en la carrera. Estas jornadas, se realizaron bajo una metodología -ZOPPpor su capacidad para promover la participación de los involucrados (GTZ, 1998). Las versiones 2.0 y 3.0 fueron evaluadas en base a un análisis comparativo con el PFF 1.0, tanto de carácter cuantitativo como cualitativo.

\section{EL PROGRAMA DE FORMACIÓN FUNDAMENTAL (PFF 1.0)}

\section{Organización, Diseño e Implementación}

Desde el año 2005, en los primeros dos años de todas las carreras de pregrado de la universidad, se comenzó a impartir un PFF con 11 módulos de 4 SCT cada uno, un conjunto de igual número de competencias generales, llamadas fundamentales, agrupadas en 3 líneas -instrumentales, interpersonales y ciudadanas-. La primera, la línea instrumental, corresponde al conjunto de competencias destinadas a habilitar al estudiante en el uso de las herramientas de trabajo intelectual indispensables para desempeñarse exitosamente en la universidad y, posteriormente, como profesionales; la segunda línea, la interpersonal, se asocia a aquellas competencias orientadas a apoyar y fortalecer el trabajo del estudiante a fin de afianzar su desarrollo personal; y la tercera, de formación ciudadana, apunta a generar en el estudiante una visión integradora del sistema socio-cultural en que se inserta (Hawes y Corvalán, 2005).

En su primera versión, el diseño del PFF se estructuró independientemente: a) de la formación básica y disciplinaria, concentrando el desarrollo de las competencias fundamentales dentro de los 4 primeros semestres de cada carrera; b) de las especificidades de cada carrera, asumiendo que los requerimientos de formación fundamental son los mismos para todas las carreras; y c) de los perfiles de entrada de los 
estudiantes de cada carrera, asumiendo por tanto que todos los estudiantes deben cursar los módulos contenidos en el PFF.

En cuanto a su implementación, el PFF se caracterizó por: a) ser resultado de una decisión estratégica de los niveles superiores de la universidad -Junta Directiva, Rectoría, Consejo Académico- conocida por los académicos, primero mediante resoluciones, y posteriormente vía Consejos de Facultad, organismos colegiados encabezados por los Decanos (UTalca, 2005)); b) aplicarse simultáneamente un mismo PFF a todos los alumnos nuevos que se matriculan desde el año 2005 en las carreras de pregrado vigentes; c) implicar la contratación de un elevado número de profesores-hora para impartir los módulos contenidos en el PFF dado que la universidad no contaba en su cuerpo docente con los profesores especialistas en el desarrollo de las competencias comprometidas en el PFF; d) marginar del PFF a los académicos de las respectivas Escuelas, lo que significó su no compromiso con el éxito del PFF; y e) dar origen a una unidad responsable de implementar el PFF dependiente directamente de la Vicerrectoría de Docencia de Pregrado.

\section{Evaluación}

A 5 años desde el inicio de su implementación, durante el año 2010, se aplicó una encuesta a los estudiantes de los niveles superiores $\left(3^{\circ}, 4^{\circ}\right.$ y $5^{\circ}$ año) de la carrera de IIE que habían aprobado la totalidad de los módulos contenidos en el PFF. Su objetivo fue identificar la contribución de cada módulo específico del PFF a la formación del estudiante y el aporte al desarrollo de las siguientes 11 competencias fundamentales explicitadas en el PFF: comunicarse eficazmente en forma oral, escrita, gráfica-icónica y de manera gestual y corporal; usar adecuadamente las herramientas metodológicas de construcción del conocimiento; lograr aprendizaje autónomo para la aplicación de metodologías innovadoras y creativas, basadas en la solución de problemas; lograr una autocrítica y autoestima equilibrada; controlar el estrés; desarrollar su proyecto profesional en concordancia con su proyecto de vida; Interactuar con otros e integrarse a redes de trabajo, amicales, sociales; comprender crítica y holísticamente el mundo y la sociedad en que vive; desarrollar y ejercer su autonomía y responsabilidad; demostrar su compromiso social; y aprender a través de la práctica de la solidaridad.

Los resultados revelaron que más del $50 \%$ de los estudiantes consideran que los módulos del PFF han contribuido poco o nada a su formación, en especial, 6 de los 11 módulos que conforman el PFF; y tampoco ha contribuido al uso adecuado de herramientas metodológicas de construcción del conocimiento, ni a controlar el estrés, ni a lograr un aprendizaje autónomo para la aplicación de metodologías innovadoras y creativas de solución de problemas. Por otra parte, los estudiantes plantearon las siguientes debilidades del PFF: excesivo número de créditos asignados al PFF, así como su concentración en los dos primeros años, etapa en la cual no se tiene cabal conciencia de su relevancia; incomprensión respecto de lo que se entiende por competencia; no adaptarse a los intereses de cada carrera; forzar la inscripción de estudiantes que posean una o más competencias fundamentales aun cuando ya las posean al ingresar a la universidad; no haberse socializado apropiadamente el PFF entre los estudiantes; haberse implantado abruptamente a nivel de toda la universidad; tener la mayoría de los docentes responsables de impartir los módulos del PFF contratos de jornada parcial o por horas; ausencia de mecanismos formales para la operacionalización, seguimiento y evaluación de los efectos del PFF en los PFB y PFD contenidos en los planes de formación de las carreras; inexistencia de una vinculación entre la unidad responsable del desarrollo del PFF y las Escuelas responsables de las carreras; y no visualizar una voluntad política de las autoridades superiores por impulsar con fuerza el modelo curricular y el PFF en particular.

No obstante todos estos alcances, sobre el $70 \%$ de los alumnos encuestados reconocen que el PFF ha contribuido al desarrollo de las competencias de interacción y de comunicación eficaz. Esta valoración se explica por tratarse de las competencias más deficitarias de los alumnos al momento de ingresar a la universidad (Schmal, 2012). Respecto de las observaciones $\mathrm{y} / \mathrm{o}$ sugerencias recogidas, ellas se concentraron en: realizar talleres o seminarios de carácter práctico orientados al desarrollo de competencias genéricas puntuales; insertar el desarrollo de las competencias genéricas dentro de los módulos orientados al desarrollo de competencias específicas de la profesión que se trate; redistribuir los módulos del PFF a lo largo del plan de formación, sobre la base de que existen competencias transversales cuyo desarrollo requieren un nivel de madurez que difícilmente se observa en los estudiantes de los primeros años; y aplicar pruebas de diagnóstico a los estudiantes que ingresan para eximirlos de cursar módulos asociados al desarrollo de las competencias que eventualmente ya posean.

Junto con la aplicación de las encuestas, se efectuó un análisis en profundidad del PFF a través de sucesivas jornadas de reflexión entre los académicos, haciendo uso de la metodología de planificación de proyectos orientada a objetivos, ZOPP (sigla correspondiente a la denominación alemana "Zielorientierte Projektplanung") de la Agencia de Cooperación Alemana (GTZ, 1998). Como resultado se obtuvo la 
identificación de los problemas que presentaba el PFF a nivel de diseño y de implementación, de los objetivos que se desean lograr, y las acciones propuestas para alcanzarlos.

El análisis y la evaluación del PFF 1.0 condujo a proponer cambios en materia de su diseño e implementación (Schmal, 2012). En el ámbito del diseño: a) reducir del tamaño del PFF (44 SCT) de modo de dejar espacio para la contextualización de las competencias implicadas y/o la formación básica y/o disciplinaria; b) clarificar el concepto de competencia, en particular su nivel de complejidad, amplitud y de desarrollo esperado; c) contextualizar las competencias genéricas a los requerimientos de cada carrera, o a grupos de carreras asociadas a una misma disciplina; d) considerar el perfil de ingreso de los estudiantes que contemple el nivel de desarrollo de las competencias genéricas que poseen; y e) desconcentrar el PFF a lo largo de las carreras en base el nivel de madurez y los requerimientos de puesta en práctica de las competencias genéricas implicadas.

En el ámbito de la implementación: a) socializar el PFF entre académicos y estudiantes de manera que los comprometa en su éxito; b) implantar el PFF gradualmente para los efectos de recoger las buenas prácticas y corregir errores; c) incorporar a la planta docente de la universidad a los profesores responsables del desarrollo de las competencias implicadas; d) crear los mecanismos institucionales formales para operacionalizar, monitorear y evaluar la continuidad del desarrollo de las competencias implicadas en el PFF en los restantes módulos del plan de formación general; e) capacitar a los profesores disponibles a fin de implicarlos en el desarrollo de las competencias implicadas; f) relacionar la unidad institucional encargada del desarrollo del PFF con las Escuelas responsables de las carreras para asegurar la continuidad del desarrollo de las competencias comprometidas; g) integrar los módulos del PFF en los módulos de formación básica y disciplinaria; y h) disponer de un claro liderazgo a nivel institucional.

\section{PFF 2.0}

\section{Organización, Diseño e Implementación}

Como consecuencia de una reflexión realizada en la totalidad de las escuelas de pregrado existentes en la universidad en torno al PFF en su versión 1.0, se resolvió su modificación para dar origen a una versión 2.0. Esta nueva versión implicó: a) reemplazar las 11 competencias fundamentales por 5 competencias fundamentales (comunicar de modo pertinente en forma oral y escrita en situaciones diversas y propias de su formación profesional; aplicar herramientas de aprendizaje autónomo como estrategia para continuar aprendiendo; lograr eficacia en el uso de habilidades sociales para establecer relaciones interpersonales adecuadas; y desempeñarse colaborativamente en equipos de trabajo, mostrando liderazgo y emprendimiento en los ámbitos económico y social); b) reducir el total de créditos del PFF, de 44 SCT a 32 SCT; y c) redistribuir el total de los 32 SCT desde los 2 primeros años a los 4 primeros años en los planes de formación de todas las carreras que contemplaba el PFF 1.0.

\section{Evaluación}

Una evaluación del PFF 2.0 en relación al PFF 1.0, da cuenta de una reducción del PFF del orden del 20\% en términos del total de créditos, al pasar de 44 a 32 SCT. Esto implica una significativa disminución de la incidencia del PFF en los planes de formación para abrir espacio a la contextualización de las competencias comprometidas en el ámbito de cada carrera específica. Por otra parte, como consecuencia de la dispersión de los módulos que conforman el PFF, desde los dos primeros años a los cuatro primeros años de cada carrera, el peso de los módulos del PFF en el primer año de cada carrera disminuye del 40 al $20 \%$ dentro de la carga académica de los estudiantes.

A ello se agrega una importante baja en el número de competencias comprometidas, disminución que es consecuencia de dos factores. Por una parte, refleja una clarificación de las competencias implicadas, en el sentido que toda competencia debe expresarse de forma tal que sea fácilmente comprensible, tanto por profesores como alumnos; por otra parte, reveló la imposibilidad de desarrollar cabalmente, en tan solo dos años las 11 competencias implicadas en el PFF 1.0. En consecuencia, los cambios recogen algunas de las observaciones más importantes que en su momento se hicieran.

Desde un punto de vista cualitativo, se observa que el PFF 2.0 abre espacio para que el desarrollo de las competencias fundamentales no sea solo responsabilidad de los módulos del PFF, sino que sea complementada por los módulos de los otros programas que conforman el plan de formación (formación básica y formación disciplinaria); y para que las competencias fundamentales vayan siendo desarrolladas a lo largo de la carrera, posibilitando que los estudiantes visualicen sus aplicaciones, con un mayor nivel de conciencia y/o madurez respecto de su razón de ser. 


\section{PFF 3.0}

\section{Organización, Diseño e Implementación}

Para pasar a una fase de consolidación del modelo de formación basada en competencias, en el año 2013, la Universidad de Talca elabora un proyecto en esa dirección, el cual es llevado a cabo bajo el alero de un convenio de desempeño de armonización curricular con el Ministerio de Educación y que la Universidad logra adjudicarse. El objetivo general establecido en el convenio consiste en adecuar el modelo educativo basado en competencias implementado por la Universidad de Talca, enfatizando la autoevaluación del proceso, la relación con el medio laboral y la vinculación con los establecimientos de educación media de la Región.

Lo expuesto implicó un análisis de los perfiles profesionales demandados por los empleadores, expresados en términos de competencias, junto con un análisis de las conclusiones emanadas de un informe de evaluación del progreso en las competencias fundamentales (Canales, 2013). Este análisis permitió observar la necesidad de integrar las competencias fundamentales con las disciplinas asociadas a las distintas profesiones, dado que la formación de competencias fundamentales no se encontraba apropiadamente contextualizada a pesar que la reducción de 12 SCT del PFF 2.0 respecto del PFF 1.0 apuntaba en esa dirección.

Como consecuencia de ello, en el año 2014 se reformuló el PFF (PFF 3.0), buscando integrar la formación fundamental con los distintas disciplinas. Lo expuesto implicó que esta nueva versión del PFF (Tabla 1) se caracterice por: a) centrarse en la formación de 3 competencias fundamentales; b) estructurarse en 8 módulos con un total de $24 \mathrm{SCT}$; y c) asignarse 8 SCT a las escuelas responsables de las carreras que administran, para que las competencias fundamentales sean desarrolladas en el contexto de las correspondientes profesiones.

Tabla 1: PFF versión 3.0

\begin{tabular}{|c|c|c|c|c|}
\hline Línea & Competencia & Módulo & $S C T$ & Sem \\
\hline \multirow{3}{*}{$\begin{array}{l}\text { Formación } \\
\text { instrumental }\end{array}$} & \multirow{3}{*}{$\begin{array}{l}\text { Comunicar discursos en forma oral y escrita, } \\
\text { basándose en los recursos lingüísticos } \\
\text { académicos para desempeñarse en } \\
\text { situaciones del ámbito profesional }\end{array}$} & $\begin{array}{l}\text { Comunicación oral y } \\
\text { escrita } 1\end{array}$ & 2 & 1 \\
\hline & & $\begin{array}{l}\text { Comunicación oral y } \\
\text { escrita } 2\end{array}$ & 4 & 2 \\
\hline & & Autogestión del aprendizaje & 3 & 3 \\
\hline $\begin{array}{l}\text { Formación } \\
\text { interpersonal }\end{array}$ & $\begin{array}{l}\text { Integrar equipos de trabajo desarrollando } \\
\text { habilidades sociales y de autogestión para } \\
\text { potenciar la capacidad de crear valor desde } \\
\text { su profesión }\end{array}$ & $\begin{array}{l}\text { Trabajo en equipo y } \\
\text { desarrollo de habilidades } \\
\text { sociales }\end{array}$ & 3 & 4 \\
\hline \multirow{4}{*}{$\begin{array}{l}\text { Formación } \\
\text { ciudadana }\end{array}$} & \multirow{4}{*}{$\begin{array}{l}\text { Discernir en los ámbitos ético profesional, } \\
\text { social, cultural, ambiental y ciudadano }\end{array}$} & $\begin{array}{l}\text { Comprensión de contextos } \\
\text { sociales }\end{array}$ & 3 & 5 \\
\hline & & $\begin{array}{l}\text { Comprensión de contextos } \\
\text { culturales }\end{array}$ & 3 & 6 \\
\hline & & $\begin{array}{l}\text { Ética y Responsabilidad } \\
\text { social }\end{array}$ & 3 & 7 \\
\hline & & $\begin{array}{l}\text { Responsabilidad } \\
\text { Social }\end{array}$ & 3 & 8 \\
\hline \multicolumn{3}{|r|}{ Total SCT } & 24 & \\
\hline
\end{tabular}

\section{Evaluación}

Al cotejar el PFF 3.0, en relación a los PFF 1.0 y 2.0, se observa que: a) el PFF se reduce primero a 44 a 32 SCT, y luego a 24 SCT, implicando una reducción de casi un $50 \%$ respecto de la primera versión del PFF, lo que conlleva que el peso del PFF en los planes de formación disminuye a un 10\% o menos, según la duración de cada carrera (Tabla 2); b) el peso de los módulos del PFF en los dos primeros años de cada carrera se reduce a la mitad de la carga académica de los estudiantes, como consecuencia de la dispersión de los módulos que conforman el PFF, desde los dos primeros años a los cuatro primeros años de cada carrera (Tabla 3); c) el número de competencias comprometidas en el PFF experimenta una significativa disminución, desde 11 competencias en el PFF 1.0, a 5 competencias en el PFF 2.0, y a 3 competencias en el PFF 3.0; y d) de los 32 SCT del PFF 2.0 se traspasan 8 SCT a las Escuelas para que en los módulos de 
los planes de formación, se complemente el desarrollo de las competencias fundamentales que más requieren los estudiantes para desempeñarse satisfactoriamente en el contexto laboral y social para el que están siendo formados.

Tabla 2: Incidencia del PFF en las carreras según su duración en semestres

\begin{tabular}{|c|c|c|c|c|}
\hline Duración $\left(n^{\circ}\right.$ semestres) & Total SCT & 2005: PFF 1.0 & 2011: PFF 2.0 & 2015: PFF 3.0 \\
\hline 8 & 240 & $44(18 \%)$ & $32(13 \%)$ & $24(10 \%)$ \\
\hline 10 & 300 & $44(15 \%)$ & $32(11 \%)$ & $24(8 \%)$ \\
\hline 12 & 360 & $44(12 \%)$ & $32(9 \%)$ & $24(6,7 \%)$ \\
\hline
\end{tabular}

Tabla 3: Incidencia semestral del PFF

\begin{tabular}{|c|c|c|c|c|c|c|}
\hline \multirow{3}{*}{ Semestre } & \multicolumn{2}{|c|}{2005} & \multicolumn{2}{c|}{2011} & \multicolumn{2}{c|}{2015} \\
\cline { 2 - 7 } & $\begin{array}{c}\text { SCT del } \\
\text { PFF 1.0 }\end{array}$ & $\begin{array}{c}\text { \%SCT del } \\
\text { PFF 1.0 }\end{array}$ & $\begin{array}{c}\text { SCT del } \\
\text { PFF 2.0 }\end{array}$ & $\begin{array}{c}\text { \%SCT del } \\
\text { PFF 2.0 }\end{array}$ & $\begin{array}{c}\text { SCT del } \\
\text { PFF 3.0 }\end{array}$ & $\begin{array}{c}\text { \%SCT del } \\
\text { PFF 3.0 }\end{array}$ \\
\hline 1 & 12 & $40 \%$ & 6 & $20 \%$ & 2 & $6,7 \%$ \\
\hline 2 & 12 & $40 \%$ & 6 & $20 \%$ & 4 & $13,3 \%$ \\
\hline 3 & 12 & $40 \%$ & 4 & $13 \%$ & 3 & $10 \%$ \\
\hline 4 & 8 & $27 \%$ & 4 & $13 \%$ & 3 & $10 \%$ \\
\hline 5 & 0 & $0 \%$ & 2 & $7 \%$ & 3 & $10 \%$ \\
\hline 6 & 0 & $0 \%$ & 4 & $13 \%$ & 3 & $10 \%$ \\
\hline 7 & 0 & $0 \%$ & 2 & $7 \%$ & 3 & $10 \%$ \\
\hline 8 & 0 & $0 \%$ & 4 & $13 \%$ & 3 & $10 \%$ \\
\hline Total & 44 & & 32 & & 24 & \\
\hline
\end{tabular}

\section{RESULTADOS}

En relación a los objetivos planteados para abordar los problemas detectados en el PFF 1.0, en la Tabla 4 se puede observar que existen objetivos que en su momento fueron planteados y que el PFF 3.0, recoge totalmente (RT), no recoge (NR), o bien recoge parcialmente (RP), tanto en el ámbito del diseño como de la implementación del PFF. En la Tabla 5 se identifican las acciones propuestas durante el proceso de implementación del modelo curricular basado en competencias, y si ellas han sido incorporadas al PFF 3.0.

Tabla 4: Objetivos planteados en el ámbito del diseño y de la implementación.

\begin{tabular}{|l|c|}
\hline \multicolumn{1}{|c|}{ Objetivos planteados en el ámbito del diseño } & PFF 3.0 \\
\hline $\begin{array}{l}\text { Reducción del tamaño del PFF (44 SCT) de modo de dejar espacio para la contextualización de las } \\
\text { competencias implicadas y/o la formación básica y/o la formación disciplinaria. }\end{array}$ & $\mathrm{RT}$ \\
\hline $\begin{array}{l}\text { Clarificación en torno al concepto de competencia, en particular su nivel de complejidad, amplitud y de } \\
\text { desarrollo esperado. }\end{array}$ & $\mathrm{RP}$ \\
\hline $\begin{array}{l}\text { Contextualización de las competencias genéricas a los requerimientos de cada carrera, o a grupos de } \\
\text { carreras asociadas a una misma disciplina. }\end{array}$ & $\mathrm{RT}$ \\
\hline $\begin{array}{l}\text { Consideración del perfil de ingreso de los estudiantes que contemple el nivel de desarrollo de las } \\
\text { competencias genéricas que poseen. }\end{array}$ & $\mathrm{NR}$ \\
\hline $\begin{array}{l}\text { Desconcentración del PFF a lo largo de las carreras en base el nivel de madurez y los requerimientos de } \\
\text { puesta en práctica de las competencias genéricas implicadas. }\end{array}$ & $\mathrm{RT}$ \\
\hline \multicolumn{1}{|c|}{ Objetivos el ámbito de la implementación } & PFF 3.0 \\
\hline Socialización del PFF entre académicos y estudiantes de manera que los comprometa en su éxito. & $\mathrm{RP}$ \\
\hline Implantación gradual para los efectos de recoger las buenas prácticas y corregir errores. & $\mathrm{RT}$ \\
\hline $\begin{array}{l}\text { Incorporación a la planta docente de la universidad a los profesores responsables del desarrollo de las } \\
\text { competencias implicadas. }\end{array}$ & $\mathrm{RP}$ \\
\hline $\begin{array}{l}\text { Creación de los mecanismos institucionales formales para operacionalizar, monitorear y evaluar la la } \\
\text { continuidad del desarrollo de las competencias implicadas en el PFF en los restantes módulos del plan de } \\
\text { formación general. }\end{array}$ & $\mathrm{RP}$ \\
\hline $\begin{array}{l}\text { Capacitación a los profesores disponibles a fin de implicarlos en el desarrollo de las competencias } \\
\text { comprometidas. }\end{array}$ & $\mathrm{RP}$ \\
\hline $\begin{array}{l}\text { Vinculación de la unidad institucional encargada del desarrollo del PFF con las Escuelas responsables de } \\
\text { las carreras para asegurar la continuidad del desarrollo de las competencias comprometidas. }\end{array}$ & $\mathrm{RT}$ \\
\hline Integración de los créditos del PFF en los módulos de formación básica y disciplinaria. & $\mathrm{RP}$ \\
\hline Existencia de un claro liderazgo a nivel institucional. & $\mathrm{NR}$ \\
\hline
\end{tabular}


Tabla 5: Acciones recogidas en el PFF 3.0

\begin{tabular}{|c|c|}
\hline Acciones & PFF 3.0 \\
\hline $\begin{array}{l}\text { Los módulos deben ser adecuados y pertinentes a la } \\
\text { carrera. }\end{array}$ & $\begin{array}{l}\text { Los módulos siguen siendo comunes a todos los alumnos de la } \\
\text { universidad, no obstante que se observa un mayor esfuerzo por } \\
\text { contextualizar cada módulo a las características de cada } \\
\text { carrera; }\end{array}$ \\
\hline $\begin{array}{l}\text { La implementación y evaluación deben estar bajo la } \\
\text { supervisión de la Escuela. }\end{array}$ & $\begin{array}{l}\text { La implementación y evaluación del PFF sigue estando bajo la } \\
\text { responsabilidad de la Vicerrectoría de Pregrado, fuera de la } \\
\text { supervisión de las Escuelas. }\end{array}$ \\
\hline $\begin{array}{l}\text { Los módulos deben agregar valor a la carrera y a la } \\
\text { formación de los estudiantes. }\end{array}$ & $\begin{array}{l}\text { Aún no existen evidencias que bajo el nuevo PFF se agregue } \\
\text { valor a la formación de los estudiantes. }\end{array}$ \\
\hline $\begin{array}{l}\text { Los módulos deben contemplar pruebas de } \\
\text { diagnóstico de modo que quienes tengan las } \\
\text { competencias comprometidas puedan eximirse de } \\
\text { ellos. }\end{array}$ & $\begin{array}{l}\text { No incorpora pruebas de diagnóstico que permita identificar a } \\
\text { los estudiantes con/sin las competencias comprometidas en el } \\
\text { PFF. }\end{array}$ \\
\hline $\begin{array}{l}\text { Existan módulos con carácter electivo para que la } \\
\text { Escuela tenga algún grado de libertad de } \\
\text { agregar/eliminar módulos. }\end{array}$ & $\begin{array}{l}\text { No contempla la inclusión de módulos de carácter electivo de } \\
\text { mayor/menor interés para las Escuelas. }\end{array}$ \\
\hline $\begin{array}{l}\text { Los contenidos de los módulos deben desagregarse } \\
\text { para mejorar tópicos individuales. }\end{array}$ & $\begin{array}{l}\text { Los contenidos de los módulos se desagregan en los syllabi } \\
\text { respectivos. }\end{array}$ \\
\hline $\begin{array}{l}\text { La carga de créditos SCT debe distribuirse a través } \\
\text { de la carrera. }\end{array}$ & $\begin{array}{l}\text { La carga de créditos se encuentra distribuida a lo largo de la } \\
\text { carrera. }\end{array}$ \\
\hline $\begin{array}{l}\text { Los módulos deben dictarse en períodos cortos e } \\
\text { intensos bajo la modalidad de talleres para aprender } \\
\text { y luego aplicar. }\end{array}$ & $\begin{array}{l}\text { Los módulos siguen conservando su carácter semestral, sin } \\
\text { perjuicio que en ellos se incluyan talleres y/o que estos se } \\
\text { implementen en los espacios abiertos por la reducción del total } \\
\text { de créditos en relación al PFF original (PFF 1.0). }\end{array}$ \\
\hline $\begin{array}{l}\text { Los talleres deben ser específicos para la formación } \\
\text { y desarrollo de habilidades ejecutivas. }\end{array}$ & $\begin{array}{l}\text { Se abre la posibilidad de talleres específicos en el marco del } \\
\text { plan de formación de cada carrera. }\end{array}$ \\
\hline
\end{tabular}

\section{CONCLUSIONES}

El análisis del PFF da cuenta de un programa que recoge el perfil de los alumnos que ingresan a la Universidad de Talca, cuya gran mayoría presentan carencias en materia de competencias transversales que están siendo fuertemente demandadas por la sociedad actual y el mundo laboral, razón por la cual su concepción es muy pertinente. Sin embargo, en el ámbito de su diseño e implementación, su primera versión mostró falencias, que han sido resueltas parcialmente en versiones posteriores.

Las vicisitudes experimentadas por el PFF a lo largo de su década de existencia no han sido menores, en gran parte explicadas porque en su momento no se tenía la capacidad y experiencia que sí existe en la actualidad, y que está posibilitando efectuar los mejoramientos efectuados hasta la fecha.

Lo más relevante del nuevo PFF (3.0) es que reduce y clarifica significativamente las competencias que compromete desarrollar, y redistribuye sus módulos dentro del plan de estudios, desde los dos primeras años a los cuatro primeros, abriendo espacio a su contextualización por parte de los académicos de cada carrera y mantener la identidad común que se aspira tengan los estudiantes al momento de egresar.

Pendiente está la necesidad de: a) efectuar pruebas de diagnóstico a los alumnos que ingresan a la universidad a fin de levantar la obligatoriedad de cursar todos los módulos del PFF a quienes ya posean las competencias transversales correspondientes consideradas en el PFF; b) flexibilizar su contenido incorporando módulos de carácter electivo para que los estudiantes puedan explotar sus potencialidades; c) profundizar la socialización entre profesores y estudiantes de las virtudes de un modelo curricular basado en competencias; $y$ d) fortalecer la institucionalidad de apoyo a la implementación del modelo.

\section{REFERENCIAS}

Agencia de Cooperación Alemana (GTZ). Planificación de proyectos orientada a objetivos (ZOPP), orientaciones para la planificación de proyectos y programas nuevos y en curso (1998). Disponible en http://www.campo-latino.org/paginas/descargas/Documentos/GTZ_ZOPP-sp.pdf Acceso: 20 de abril (2010) 
Alonso, L.; C. Fernández y J. Nyssen, J. (Agencia Nacional de Evaluación de la Calidad y Acreditación), El debate sobre las competencias. Una investigación cualitativa en torno a la educación superior y el mercado (2009). Disponible en

http://www.aneca.es/content/download/10356/115906/file/publi_competencias_090303.pdf Acceso: 10 de Febrero (2013).

Aristimuño, A., Las competencias en la educación superior: ¿demonio u oportunidad?, Departamento de Educación, Universidad Católica de Uruguay (2004). Disponible en

http://www.cedus.cl/files/competencia_aristumuño.pdf Acceso: 24 de agosto (2014)

Brunner, J.J., Educación Superior en Chile: instituciones, mercados y políticas gubernamentales (19672007), Universidad Diego Portales, Santiago-Chile (2009)

Canales, T., Informe de Evaluación del Progreso de la Cohorte 2010, Centro de Psicología Aplicada, Facultad de Psicología-Universidad de Talca, Talca-Chile (2013)

Carmona, R., Toro, I. y Riascos, J.A., Caracterización de las Instituciones de Educación Superior (IES) en Chile: una aproximación a un modelo futuro de universidad (Principales reformas que se han presentado en el modelo de educación superior en Latinoamérica impactando en la mercantilización de las universidades). Revista Educación de la Universidad de Costa Rica, 38(2), 37-50 (2014)

CASEN. Educación encuesta Caracterización Socioeconómica Nacional, Ministerio de Planificación, Chile (2009). Disponible en http://www.mideplan.cl/casen2009/casen_educacion.pdf Acceso: 9 agosto (2014)

Castells, M., La sociedad red, vol 1., Ed. Alianza, España (2005)

Corominas, E. Competencias genéricas en la formación universitaria, Revista de Educación, 325, 299-321 (2001)

Faúndez, F.; Gutiérrez, A.; y Ponce, M., Desarrollo institucional del currículo basado en competencias: el caso de la Universidad de Talca, Documento de Trabajo-Universidad de Talca, Talca-Chile, pp. 50 (2009)

González, J.; Wagenaar, R.; y Beneitone P., Tuning-América Latina: Un proyecto de las universidades. Revista Iberoamericana de Educación, 35, 151-64 (2004). Disponible en http://www.rieoei.org/rie35a08.pdf Acceso: 20 abril (2014)

Hawes G.y Corvalán, O., Competencias fundamentales en programas de formación profesional de pregrado de la Universidad de Talca. Programa MECESUP TAL 0101, Universidad de Talca (2005)

Hernández, R., Fernández, C. y Baptista, P., Metodología de la Investigación, México: MacGraw-Hill (2006)

Jonnaert, P., Competencias y socioconstructivismo. Nuevas referencias para los programas de estudios. Texto de apoyo a la Segunda Conferencia Anual de Inspectores de la Enseñanza Media, Bobo Dioulasso, Burkina Faso, 18-22 de diciembre; 2001. Disponible en

http://psicologiaporlavida.blogspot.com/2011/11/competencias-y-socioconstructivismo.html Acceso: 20 de febrero (2014)

Kallioinen, O. Defining and comparing generic competences in higher education. European Educational Research Journal, 9(1), 56-68 (2010)

Martí, J.J., Martí, M., Vargas O. y Moncayo, J., Reflexión sobre los discursos en educación superior. Revista de la Educación Superior, 43(4), 33-55 (2014)

Mertens, L., Competencia laboral: sistemas, surgimiento y modelos. Cinterfor, Montevideo-Uruguay (1996). Disponible en

http://acreditacion.unillanos.edu.co/contenidos/4_jornada_pedagogica_2008/mertens_librocompleto.pdf

Acceso: 23 de abril (2014)

Molina, J., Educación pública, autonomía universitaria y cambio político: notas para el análisis del movimiento universitario en Chile, 2011. Revista Historia de la Educación Latinoam., 15(21), 263-282 (2013) 
Montero, I. y León, O. Clasificación y descripción de las metodologías de investigación en Psicología, Revista Internacional de Psicología Clínica y de la Salud, 2(3), 503-508 (2002)

Moyano, E., Vásquez, M. y Faúndez, F., El modelo educativo de la Universidad de Talca. Ed. Universidad de Talca, Chile (2012). Disponible en http://vrdp.utalca.cl/docs2014/libro_moyanovasquezfaundez2012.pdf Acceso: 20 de diciembre (2014)

Norton, R. A tool for developing curricula, Vocational Education Journal, 62(3), 5-15 (1987)

Olavarría, M. y Allende, C., Endeudamiento estudiantil y acceso a la educación superior en Chile. Revista Española de Investigaciones Sociológicas (REIS), 141, 91-112 (2013). Disponible en http://www.reis.cis.es/REIS/PDF/REIS_141_041358269547179.pdf Acceso: 10 de noviembre (2014)

Palominos, F.E., Mendez, M.A. y Barrera, R.A. Sistema de Perfeccionamiento Orientado a Competencias para Docentes de la Educación Superior, Formación Universitaria, 7(3), 11-22 (2014)

Peluffo, M. y Knust, R., Aproximación a la educación universitaria por competencias en América Latina: ¿Una "fata morgana" o un modelo factible para la realidad latinoamericana? (2009). Disponible en http://mt.educarchile.cl/MT/jjbrunner/archives/2010/03/aproximacion_a.html Acceso: 26 de octubre (2014)

Rojas, A..y Tarud, J.A., La Innovación Curricular: factores que la determinan y modelos de implementación, Universidad de Talca, Chile (2004)

Ruiz G., El enfoque de la formación profesional en torno a la generación de competencia: ¿ejercicio impostergable o "lo que sucedió a un rey con los burladores que hicieron el paño"?, Estudios Pedagógicos, 35(1), 287-299 (2009)

Salas, M. Do higher education institutions make a difference in competence development? A model of competence production at university. Higher Education, 68(4), 503-523 (2014)

Savaneviciene A., Rutelione, A. y Ciutiene, R. Crucial Transversal Competences in the Changing Environment: Case of the European SMES Managers. Economics and Management, 19(1), 100-108 (2014)

Schmal, R., Reflexiones en torno a un Programa de Formación en Competencias Transversales en Ingeniería. Revista de Ciencia, Docencia y Tecnología, Entre Ríos-Argentina, 44, 239-262 (2012)

Sicilia, M.A. How should transversal competence be introduced in computing education?, SIGCSE Bulletin, 41(4), 95-98 (2009)

Silva, A., Competencias blandas en la formación de ingenieros, XXI Congreso Chileno de Educación en Ingeniería, Universidad de Chile, Santiago-Chile (2007). Disponible en

http://www.ici.ubiobio.cl/ccei2007/papers/54.pdf Acceso: 20 de abril (2014)

Tardif, J. Desarrollo de un programa por competencias: de la intención a la puesta en marcha. PROFESORADO, Revista de Curriculum y Formación de Profesorado, 12(3), 1-16 (2008). Disponible en http://www.ugr.es/ recfpro/rev123ART2.pdf Acceso: 30 de marzo (2015)

Tobón, S., El enfoque complejo de las competencias y el diseño curricular. Acción Pedagógica, 16, 14-28 (2007). Disponible en http://es.scribd.com/doc/50453411/Competencias-Tobon Acceso: 4 de enero (2015)

Tobón, S. La formación basada en competencias en la educación superior: el enfoque complejo. ESCOE ediciones, Bogotá-Colombia (2008)

Universidad de Talca., Perfil genérico y la estructura curricular del grado de licenciado y del título profesional. Resolución № 082 del 18 de enero (2005)

Villa, A. y Poblete, M. Evaluación de competencias genéricas: principios, oportunidades y limitaciones. Bordón 63(1), 147-170 (2011) 
Villarroel, V. y Bruna D., Reflexiones en torno a las competencias genéricas en educación superior: Un desafío pendiente. Psicoperspectivas, 13(1), 23-34 (2014).

Yanaze, L.K. y De Deus, R., Transversal competencies of electrical and computing engineers considering market demand, Frontiers in Education Conference (FIE), IEEE, 22-25 octubre, Madrid-España (2014). Disponible en http://ieeexplore.ieee.org/stamp/stamp.jsp?tp=\&arnumber=7044169

Yániz, C. Las competencias en el currículo universitario: implicaciones para diseñar el aprendizaje y para la formación del profesorado, Revista de Docencia Universitaria, 6(1), 1-13 (2008)

Yániz, C. Las competencias genéricas como finalidad educativa en Competencias genéricas en Educación Superior, Villardón, L. Ed. Narcea, Madrid-españa, 13-24 (2015)

Zúñiga, M., Poblete, A. y Vega A., El desarrollo de competencias en un contexto de aseguramiento de calidad, en Diseño curricular basado en competencias y aseguramiento de la calidad en la educación superior, CINDA, Santiago-Chile (2008) 